\title{
Tilingsearch.org: A searchable repository of Islamic geometric patterns
}

\author{
Brian Wichmann \\ Woking, UK \\ http://www.tilingsearch.org \\ Brian.Wichmann@bcs.org.uk
}

\section{INTRODUCTION}

In 2007, a website was started which aimed to collect all Islamic geometric design having a mathematical basis. After 13 years, the collection is essentially finished, and this paper summarises the conclusions. With over 1,000 Islamic geometric patterns, no other collection of this size is known.

\section{STARTING}

An initial decision was to use MySQL as an underlying technology to support the work. This decision proved to be very effective in allowing searching and analysis of the available material. For the details, see Wichmann (2019).

Two vital aspects enabled the collection to start:

(i) The website by David Wade which provide a source of photographs (Wade 2020) from which to encode the design using simple geometry; see Wichmann (2017) for details.

(ii) Contacting Tony Lee who had started analysing Islamic designs many years before me and who had a wealth of experience to impart (as well as web pages).

\section{COLLECTION METHOD}

The potential problem of copyright was avoided by only referencing photographs and displaying only the resulting analysis via PDF. The main source of data was the photos in David Wade's website (Wade 2020) and books in the National Art Library, see the list of over 200: Tiling References (Wichmann 2020).

From the photographs, an analysis was made using simple geometry, see Wichmann \& Wade (2017, chapters 7-9). The analysis provides the means of producing a suitable graphic which is displayed via small GIFs or high-resolution PDF.
The small GIFs are used to display groups of patterns, while to PDF is used to give the full details. Taking an informal photo of illustrations in books is essential to allow for analysis of the design.

The collection was expanded by inspecting relevant books, and by contributions of photos and references by more than 60 other people interested in Islamic patterns.

Although the data on the website is about $400 \mathrm{Mb}$, the entire system is about $13 \mathrm{~Gb}$. The extra material is the SQL, software and (mainly) documentation.

\section{PROBLEMS}

Four issues are considered in the subsections below.

\subsection{Incomplete source data}

Photos of Islamic building are typically panoramas with low resolution for actual patterns. Hence David Wade's site is exceptional in providing good quality photos of patterns. In some cases, books only give a drawing so that the website version may be dependent on such drawings. Jules Bourgoin has a further problem in not giving a source which implies a photo could not be even be obtained (Bourgoin 1879; Lee 2019).

Books were used, mainly from the National Art Library, but sometimes the location was often not given. Internet source of photos was even less reliable in giving a location.

\subsection{Complete mathematical analysis}

In many cases, an Islamic geometric pattern can be analysed in a manner that gives no doubt as to the intention of the design. Examples are given in Wichmann \& Wade (2017, chapters 7-10). As an example of a complex pattern with a relatively 
simple mathematical analysis see Figure 1. The main part of this pattern outside the central star uses the tiles defined in Wichmann \& Wade (2017, chapter 9). The tricky computation is the shape of the black tiles surround the main star; see Wichmann \& Wade (2017, section 10.2) for the details.

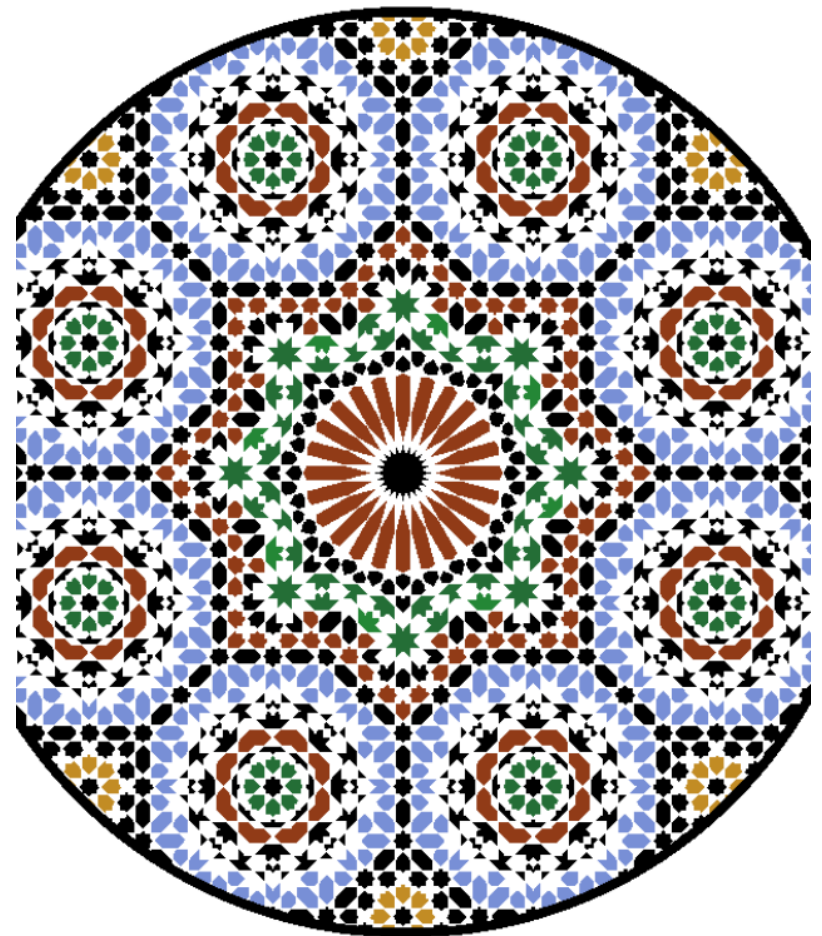

Figure 1: Neijarine Palace, Fez, Morocco.

Pattern uses 465 polygons, with over 3,000 edges (http://www.tilingsearch.org/HTML/data196/N4.html).

A potential difficulty in the design analysis is illustrated by the pattern in Wichmann \& Wade (2017, sections $16.1 \& 16.2$ ). The first analysis produces a purely mathematical analysis (as on the website), while the second produces what is supposed to the same pattern by a manual drawing. The problem is that the two patterns look similar but are not exactly the same. In other words, there is some uncertainty in the analysis.

With more complex patterns, a mathematical analysis becomes tedious to undertake and does not give any useful insight into the design process (which was almost certainly artistic rather than mathematical).

Rather than exclude such patterns, it was decided to allow patterns without a mathematical analysis, typically drawn manually using Inkscape. This decision allowed many more patterns to be included but makes some aspect of the system more complex (especially searching).

As an example, based on a photo by me, consider Figure 2.
This pattern has 16-pointed, 7-pointed and 12pointed stars. There are many patterns with either 16, 12 or 7-pointed stars, but rarely all three. In such a case, assuming both stars are regular, one would expect the angles of the tile to be multiples of $\pi /(7 * 3 * 16)$ or about 0.53 degrees. Hence although a mathematical analysis would be possible, it would not add anything to the understanding of the pattern. In this case, consider the triangle composed of a horizontal line joining the two centres of the 7-stars with two lines to the central main star. The angle of the triangle at the central star is $22.5^{\circ}$, while the angle to the horizontal line should be $3 * 180 / 7=77.14 \ldots \circ$. Hence not an exact fit, which is overcome with manual methods by making small adjustments. Of course, those adjustments could be made to a mathematical analysis....

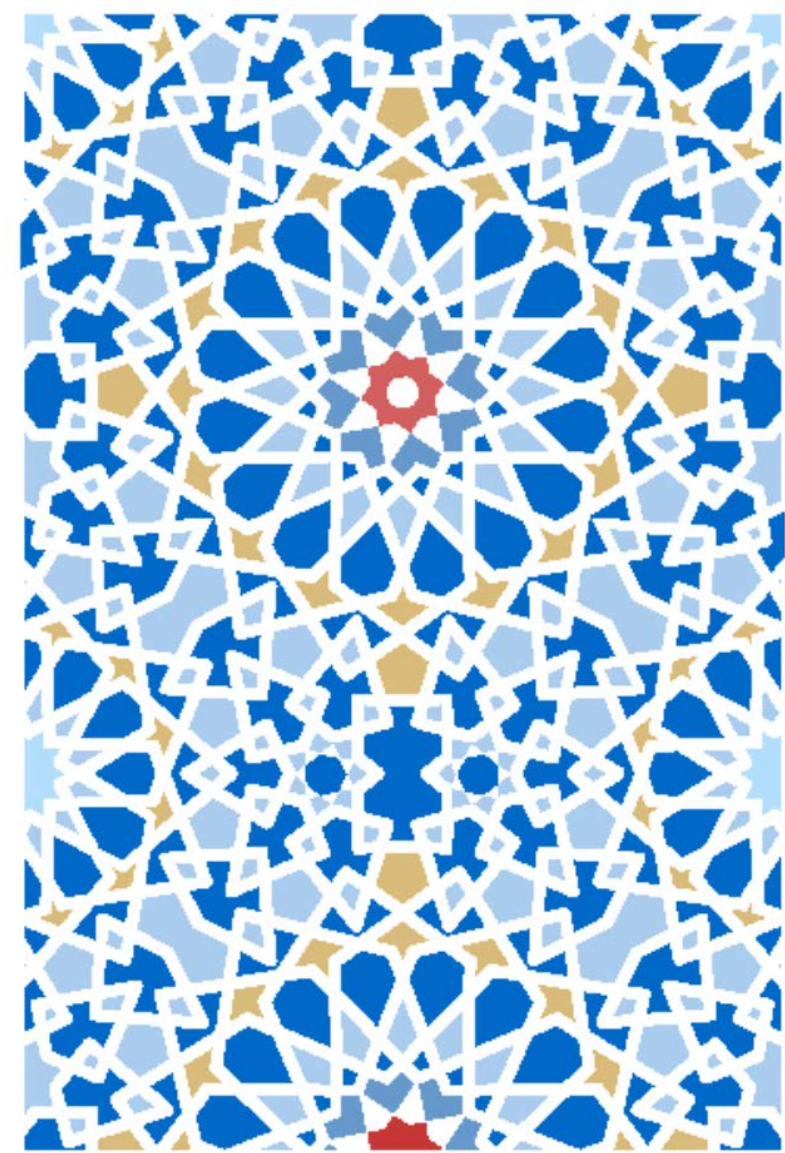

Figure 2: Regent's Park Mosque (by Tony Lee). Drawn using Inkscape, a tool based upon SVG. Shows ornamentation inside star, not usually shown on the website

(http://www.tilingsearch.org/HTML/dataO/REG1.html).

For this pattern, the existence of the 7-pointed, 12pointed and 16-pointed stars is in the database, but the angles of the stars are not given. In other words, some properties are undefined for nonmathematical patterns. 


\subsection{Documentation}

At first, the documentation consisted of a file containing $x, y$ numerical data to produce the graphic and a possible photo. It was soon realised that a more comprehensive system was needed. Some software was written to do this. The essential elements were the ability to produce the $x, y$ vector data in symbolic format, say $A^{\star} \sin (30),-A^{\star} \cos (30)$, and also a means of producing a single PDF file with all the essential documentation. Although these documentation files are not on the web, a single example is shown in The construction of Alcazar, Cuarto del Principe (Wichmann 2016).

The documentation could all have been in XML format. The only project in which I was involved decided not to use XML, so I have no experience using this. The output PDF is not in PDF-A format due to the links to other parts of the system and reference to external links with David Wade's pictures and the $V \& A$ website.

\subsection{The future}

It is hoped that a permanent site will be found which could therefore continue for longer than my involvement.

Currently, it is apparent that the index to the location of Islamic sites may contain errors. Due to the variation in the names used, this issue is not easy to resolve. The websites Archnet (2020) and Geonames (2020) both provide facilities which could be useful to clarify the problems. This area is under investigation. It might well be possible to add location maps via the Geonames system.

\section{CONCLUSIONS}

The project was an ideal retirement activity, since finishing each pattern provided some satisfaction. Stating that the project was 'complete' was a subjective judgement, since adding a small variant of an existing pattern is always possible. The pattern in Figure 2 was added in part because of the excellent colour; details of the craftsmen who produced this are not known.

After every new batch of patterns, a new release was produced since the additional checks for a release was time-consuming. The use for SQL was a success, the usual rebuild of the entire system only takes about three minutes.

Contact with people round the world via the Internet was a useful addition to the information gained from books. The work would have been barely possible without Tony Lee, David Wade, and the other 60 people who made contributions (see the manual on the website http://www.tilingsearch.org for the complete list).

\section{ACKNOWLEDGEMENTS}

This paper was edited and formatted with assistance from Jonathan Bowen. See also Wichmann \& Lee (2013).

\section{REFERENCES}

Archnet (2000) URL: http://www.archnet.org

Bourgoin, J. (1879) Les Eléments de l'art arabe: le trait des entrelacs, Paris. (Patterns available as a Dover reprint.)

Geonames (2020) URL: http://www.geonames.org

Lee, T. (2019) Possible Sources for Bourgoin (1879) "Le Trait des Entrelacs". URL: http://www.tilingsearch.org/BourgoinSources.pdf

Wade, D. (2020) Patterns in Islamic Art. URL: http://www.patterninislamicart.com

Wichmann, B. and Lee, T. (2013) Analysis and Synthesis of Islamic Geometric Designs. In K. Ng, J. P. Bowen, and S. McDaid (eds.), EVA London 2013: Electronic Visualisation and the Arts, pp. 258-259. BCS, Electronic Workshops in Computing. DOI: 10.14236/ewic/EVA2013.54

Wichmann, B. (2016) The construction of Alcazar, Cuarto del Principe. Tilingsearch.org, 16 September. URL: http://www.tilingsearch.org/ltxALZ1.pdf

Wichmann, B. (2019) SQL and Islamic Geometric Design. Nexus Network Journal, 21, pp. 669-680. DOI: https://doi.org/10.1007/s00004-019-00439-2

Wichmann, B. (2020) Tiling References. URL: http://www.tilingsearch.org/outrefs.htm/

Wichmann, B. and Wade, D. (2017) Islamic Design: A Mathematical Approach. Birkhäuser. DOI: 10.1007/978-3-319-69977-6 